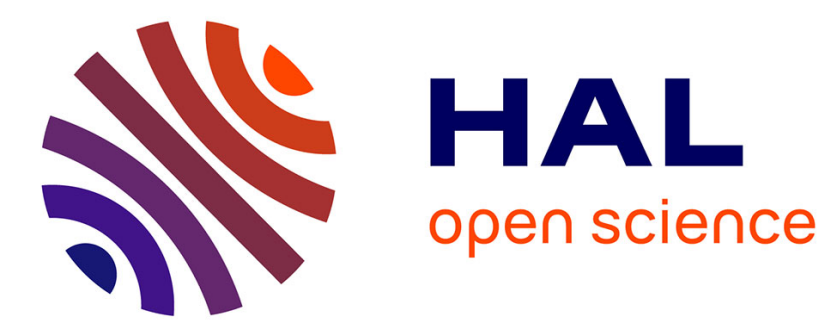

\title{
Statistics of the optical intensity of a chaotic external-cavity DFB laser
}

Nianqiang Li, Byungchil Kim, A. Locquet, Daeyong Choi, Wei Pan, D. S Citrin

\section{- To cite this version:}

Nianqiang Li, Byungchil Kim, A. Locquet, Daeyong Choi, Wei Pan, et al.. Statistics of the optical intensity of a chaotic external-cavity DFB laser. Optics Letters, 2014, 39 (20), pp.5949. 10.1364/OL.39.005949 . hal-03079704

\section{HAL Id: hal-03079704 https://hal.science/hal-03079704}

Submitted on 17 Dec 2020

HAL is a multi-disciplinary open access archive for the deposit and dissemination of scientific research documents, whether they are published or not. The documents may come from teaching and research institutions in France or abroad, or from public or private research centers.
L'archive ouverte pluridisciplinaire HAL, est destinée au dépôt et à la diffusion de documents scientifiques de niveau recherche, publiés ou non, émanant des établissements d'enseignement et de recherche français ou étrangers, des laboratoires publics ou privés. 
To be published in Optics Letters:

Title: $\quad$ Statistics of the Optical Intensity of Chaotic External-Cavity DFB Lasers Authors: Nianqiang Li, Byungchil Kim, Alexandre Locquet, Daeyoung Choi, Wei Pan, and David Citrin

Accepted: 14 September 2014

Posted: 15 September 2014

Doc. ID: 220059 


\title{
Statistics of the optical intensity of a chaotic external-cavity DFB Laser
}

\author{
Nianqiang Li, ${ }^{1}$ Byungchil Kim, ${ }^{2,3}$ A. Locquet, ${ }^{3,2}$ Daeyong Choi, ${ }^{2,3}$ Wei Pan, ${ }^{1}$ and D. S. Citrin ${ }^{2,3, *}$ \\ ${ }^{1}$ Center for Information Photonics and Communications, Southwest Jiaotong University, Chengdu, 610031 China \\ ${ }^{2}$ Georgia Institute of Technology, School of Electrical and Computer Engineering, Atlanta, Georgia 30332-0250 USA \\ ${ }^{3}$ UMI 2958 Georgia Tech-CNRS, Georgia Tech Lorraine, 2 Rue Marconi F-57070, Metz, France \\ *Corresponding author: david.citrin@ece.gatech.edu
}

\begin{abstract}
We study experimentally and theoretically the first- and second-order statistics of the optical intensity of a chaotic externalcavity semiconductor DFB laser in fully-developed coherence-collapse. The second-order statistic is characterized by the autocorrelation, where we achieve consistent experimental and theoretical results over the entire parameter range considered. For the first-order statistic, we find that the experimental probability-density function is significantly more concentrated around the mean optical power and robust to parameter changes than theory predicts. (C) 2014 Optical Society of America

OCIS Codes: (140.3490) Lasers, distributed-feedback; (140.5960) Semiconductor lasers; (190.3100) Instabilities and chaos.
\end{abstract}

The dynamics of semiconductor lasers with delayed optical feedback, [external-cavity semiconductor lasers (ECLs)], are of great interest [1-9]. Much work has been devoted to elucidating the nonlinear dynamics of ECLs [10-12]. The Lang-Kobayashi (LK) model is useful to understand feedback-related dynamics of ECLs [13], though it is not successful in accounting for all observations; it does not consider spatial dimensions and thus does not describe the overlap of the optical mode with the gain medium nor does it account for multiple round-trips in the external cavity (EC). Still, LK accounts qualitatively and sometimes quantitatively [14] for many experimentally observed dynamical regimes [1] as well as certain dynamical tendencies depending on injection current $J$, cavity length $L$, and feedback rate [12]. In view of LK's successes, it is important to determine how well it reproduces statistical properties associated to ECL dynamics. In addition to being an important way to characterize chaotic processes, knowledge of the statistics is fundamental in random-bit generation (RBG) applications as it determines both the random-bit rate and the post-processing techniques that should be used to generate random bits $[15,16]$. Due to the complexity of the dynamics and the multidimensional parameter space, a systematic statistical comparison of observed behaviors and that predicted by LK model is called for.

Previous studies of the statistics of the time-dependent intensity $I(t)$ focused on the low-frequency fluctuations (LFF) regime in single- and multi-longitudinal mode lasers [17-19], typically seen near threshold $J_{t h}$ for low-tomoderate feedback [20-24]. LFF is characterized by rapid $(<1 \mathrm{~ns}) I(t)$ dropouts followed by a slow (several ns) recovery during which the laser pulses, and can be explained as chaotic itinerancy with drift [24, 25]. Pulsing occurs not only in LFF but also for slightly higher $J$ [25], as well as determines to some extent the statistics of $I(t)$ [26]. At even larger $J$, a chaotic regime of fully-developed coherence-collapse (CC) can be observed, in which $I(t)$ typically undergoes sub-ns fluctuations around its mean, but seldom reaches zero and without dropouts.
Experimental studies of the probability-density function (PDF) of $I(t)$ in LFF with high-bandwidth apparatuses show, for truly single-longitudinal mode emission, PDFs peaked just above the spontaneous-emission level and decreased monotonically with increasing $I(t)$ [17, 18]. These investigations also show that numerical simulation of LK can reproduce the main features of the experimental PDFs.

While the statistics of $I(t)$ in LFF have been studied and compared to LK, statistical studies for CC are sparse $[27,28]$. It is known that LFF can occur slightly above or below the threshold current $J_{t h}$ of the solitary laser [11]; the associated dropouts usually rapidly disappear in experiment at $J$ just a few percent above $J_{t h}$ as CC is entered. In this Letter, we explore the agreement between experimental and numerical first- (FO) and second-order (SO) statistics of an ECL operated in CC. The FO statistic of the chaotic process is characterized by means of the PDF of $I(t)$ while its SO statistic is studied by means of the autocorrelation function (ACF). Specifically, the injection current is set in the range $J=1.4 J_{t h} \sim 2.4 J_{t h}$. We find that a good fit between experimental and theoretical ACFs but significant discrepancy between the PDFs.

The experimental setup for an EC DFB laser was similar to that in [12]. It consists of the intrinsically single-longitudinal-mode MQW InGaAsP DFB laser that oscillates at $1550 \mathrm{~nm}$ and has maximum $\mathrm{cw}$ power of 15 $\mathrm{mW}$. The free-running $J_{t h}$ is $\sim 9.27 \mathrm{~mA}$. The EC length $L$ is $65 \mathrm{~cm}$ (EC round-trip time $\tau=4.3 \mathrm{~ns}$ ). With the help of a motorized piezo-actuation stage, the angle of the quarterwave plate (QWP) is controlled in small steps. The maximum feedback (experimental feedback rate $\eta=1.0$ ) is reached when the QWP is such that the polarization is not subject to any rotation. In our experiment, $\sim 20 \%$ of the optical power is fed back onto the collimating lens for $\eta=1.0$. To detect $I(t)$, the output of the DFB laser is first coupled to a $12-\mathrm{GHz}$ photodiode, and then captured by a $12-\mathrm{GHz}$ oscilloscope with $40 \mathrm{GS} / \mathrm{s}$ sampling rate.

LK consists of rate equations for the slowly varying envelope of the complex electric field $E$ and carrier density 
$N$ [13, 29]. Parameters are defined in [29]. Namely, $G(t)=g\left[N(t)-N_{0}\right]$ is the optical gain, with $g$ the differential gain coefficient, $N_{0}$ the carrier density at transparency, $\alpha$ the linewidth-enhancement factor, $\omega_{0}$ the angular frequency of the solitary laser, $\tau_{p}$ the photon lifetime, $\tau_{e}$ the carrier lifetime, $k_{f}$ the theoretical feedback rate, $\tau$ the feedback time delay, and $q$ the pump factor $\left(J=q J_{t h}\right)$. We take $\alpha=3, \tau_{p}=2.65 \mathrm{ps}, \tau_{e}=2.5 \mathrm{~ns}$, $g=2.5 \times 10^{-8} \mathrm{ps}^{-1} \quad, \quad N_{0}=1.3 \times 10^{8} \quad, \quad \omega_{0} \tau=0 \quad$, giving $J_{\text {th }} \approx 9.27 \mathrm{~mA}$, corresponding to $J_{t h}$ of the DFB laser. The delay is not varied here and is set equal to the experimental value; $k_{f}$ and $q$ are varied according to experimental conditions. Noise is not present in the simulations presented here as we found that moderate noise does not significantly change the statistics for the relatively large $J$ considered here. When $\eta=1$, we take $k_{f}=25 \mathrm{~ns}^{-1}$ as will be shown below.

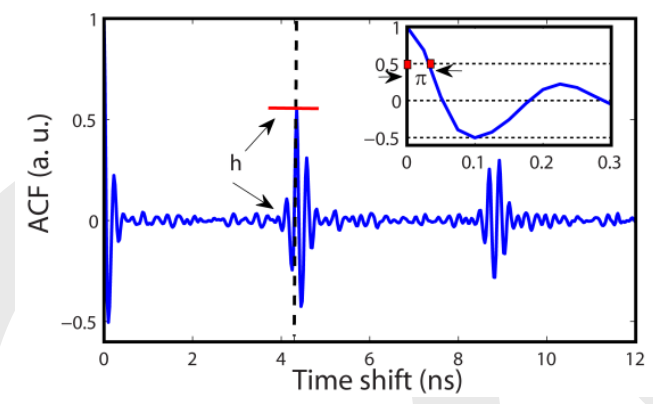

Fig. 1. ACF for $\tau=4.3 \mathrm{~ns}, J=1.4 J_{t h}$ and $k_{f}=18 \mathrm{~ns}^{-1}$. The vertical dashed line indicates $\tau$. The inset is the ACF around zero delay.

The statistics are studied by the PDF of $I(t)$, characterizing the FO statistic, and the ACF for the SO statistic by quantifying the correlation between two intensity values separated in time [29]. We first focus on the comparison between the experimental and numerical ACFs as functions of $\eta\left(k_{f}\right)$ for four values of $J$ in CC: $1.4 J_{t h}, 1.7 J_{t h}, 2.0 J_{t h}$, and $2.4 J_{t h}$. To mimic the filtering imposed by the photodiode and oscilloscope, the theoretical time traces were subsequently filtered using a cascade of two third- and ninth-order Butterworth filters with a bandwidth of $12 \mathrm{GHz}$, corresponding to frequency responses of the photodiode and oscilloscope used.

To help interpret the results, a typical ACF of $I(t)$ obtained from the LK equations is shown in Fig. 1 with $J=1.4 J_{t h}$ and $k_{f}=18 \mathrm{~ns}^{-1}$. A peculiarity of delay systems is the existence of a local maximum $h$ of the ACF near the EC delay (vertical dashed line) [29]. This gives useful information on the chaotic dynamics as changes in its value can be used to identify transition from weak to strong chaos [30-32].

Figure 2 (a) shows theory and (b) experiment for $h$ at various $J$ and $\eta\left(k_{f}\right)$ : at all $J$ shown, as $\eta\left(k_{f}\right)$ increases, $h$ first falls, becoming a dip for a critical intermediate $\eta$ or $k_{f}$, and then increases. We see that theory is in good agreement with experiment, both in terms of curve shape and its variation with increasing $J$ (dip moves to larger $\eta$ or $k_{f}$ as $J$ increases); it also suggests that the theoretical parameters are reasonable to reproduce experiment. These results also confirm numerical results in [29] in which the identifiability of the EC delay based on an analysis of $I(t)$ was explored. Additionally, it is interesting to investigate the half-width $\pi$ of the zeroth ACF peak that is a standard indicator of the correlation decay time (inset, Fig. 1), and is also a good indicator of the bandwidth of chaotic processes. In chaosbased RBG, sampling typically cannot be performed faster than this decorrelation time, thus restricting the sampling rate [4-6]. In chaos-based communications, the decorrelation time sets an upper bound in the speed at which a chaotic carrier can be modulated [16]. Fig. 2(c) shows theory and (d) experiment for $\pi$ for various $J$ and $\eta\left(k_{f}\right)$. At all $J$ shown, experiments and simulations show good agreement as $\pi$ decreases monotonically when increasing $\eta\left(k_{f}\right)$. Moreover, in both cases, larger $J$ results in smaller $\pi$, leading to a larger chaotic bandwidth.

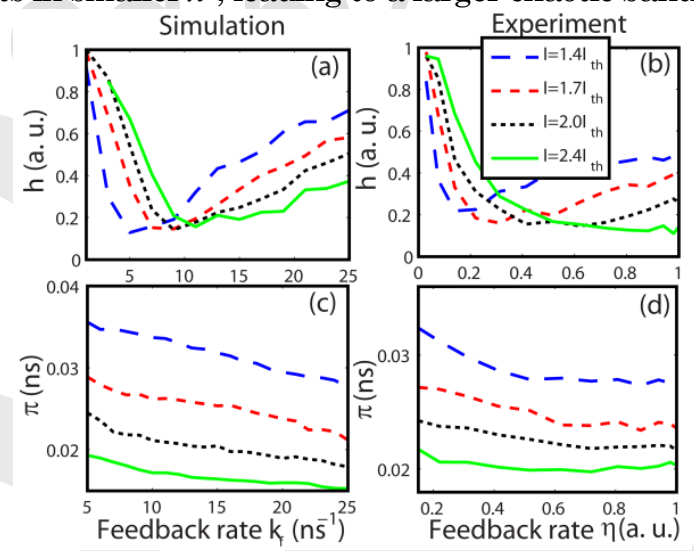

Fig. 2. Numerical (a, c) and experimental (b, d) results for $h$ and $\pi$ at four $J:\left(1.4 J_{t h}, 1.7 J_{t h}, 2.0 J_{t h}\right.$, and $\left.2.4 J_{t h}\right)$.

We consider the FO statistic of $I(t)$, characterized by its PDF. Fig. 3 shows numerical and experimental PDFs. Each is derived from $4 \times 10^{4}$ samples of $I(t)$ separated by $25 \mathrm{ps}$. The horizontal axis is scaled to its average value $\langle I\rangle$, while the PDFs are plotted as functions of $k_{f}(\eta)$ for four $J_{\text {well }}$ above $J_{t h}\left(1.4 J_{t h}, 1.7 J_{t h}, 2.0 J_{t h}\right.$, and $\left.2.4 J_{t h}\right)$; also, for all $k_{f}(\eta)$ considered the ECL operates in CC.

Figures 3(e)-(h) show the measured PDFs. These are peaked near $\langle I\rangle$ and fall off both at low and high $I(t)$ over a wide parameter range. The shape of the experimental PDFs is well fitted for all $\eta$ and $J$, by an exponentially decreasing function of the normalized intensity, around a maximum located at $\langle I\rangle$, but with different decay rates on each side of the maximum. This exponentially decreasing shape is consistent with our independent experimental work utilizing a different DFB laser and fiber-optic components (not shown) [33], as well as with various published PDFs obtained from chaotic DFB lasers with delayed optical feedback [34-36].

Figures 3(a)-(d) show the corresponding PDFs of the filtered time traces obtained from the LK equations. As in experiment, the numerical PDFs appear to be unimodal for all $k_{f}$ and $J$. Another similarity with experiments is 
that at high $J$, the PDFs exhibit a maximum near $\langle I\rangle$ [Figs. 3(b)-(d)].

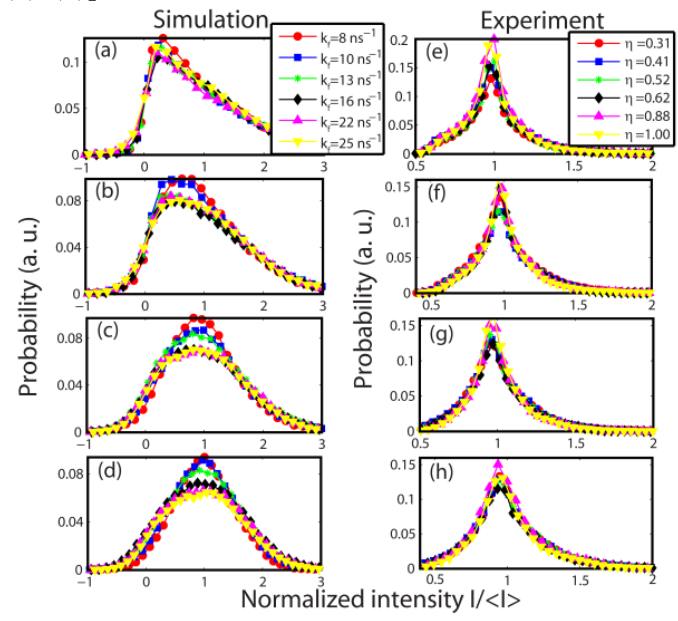

Fig. 3. Numerical (a-d) and experimental PDFs (e-h) of $I(t)$ for various $k_{f}(\eta)$ and $J: 1.4 J_{t h}(\mathrm{a}, \mathrm{e}), 1.7 J_{t h}(\mathrm{~b}, \mathrm{f}), 2.0 J_{t h}(\mathrm{c}, \mathrm{g})$, and $2.4 J_{t h}(\mathrm{~d}, \mathrm{~h})$.

Significant differences also exist between experimental and numerical PDFs. Simulated PDFs show, at all $J$, more dispersion than experiments. We see that the experimental PDF shape, at $J$ considered, is weakly influenced by $\eta$. By contrast, simulations show a change in the dispersion of the PDFs with increasing $k_{f}$. Moreover, the numerical PDFs tend toward Gaussian for large $J$, while the experimental ones are exponential at all $J$. Also, the numerical PDFs in Fig. 3(a), for low $J$, are peaked at low $I(t)$, while the corresponding experimental PDFs are centered close to $\langle I\rangle$.

The discrepancies in terms of the location of the PDF maximum may be associated with stronger pulsing in the LK model [26], at low $J$, compared to experimental results [Fig. 3 (a), (e)]. It has been shown theoretically and experimentally in truly single-mode ECLs $[17,18]$ that in LFF, the PDFs peak close to zero $I(t)$. This shape is attributed to the pulsation of the $I(t)$ dynamics and thus to the associated larger dwell time of $I(t)$ near zero, between two pulses. These pulsations are predicted by the LK model [24], and have been observed experimentally in LFF but also for $J$ larger than those leading to LFF [25]. The origin of the discrepancy is illustrated by Figs. 4 and 5 , which represent experimental and filtered simulated intensities. We observe that $40 \%$ above $J_{t h}$, the simulated $I(t)$ still exhibits a clear tendency to pulse and to keep a small value between pulses. This is not the case of the experimental time series, consistent with the differences in maximum locations in the PDFs. At larger $J$, both the numerical [Figs. 4(c) and 5(c)] and experimental [Figs. 4(d) and $5(\mathrm{~d})$ ] time series experience fluctuations around their averages, as reflected by the corresponding PDFs in Fig. 3.

In the LK model at low $J, I(t)$ tends to keep a close-tozero value after a pulse has occurred due to a depleted reservoir of carriers; at large $J$, the rate at which carriers are supplied by the current source is larger and thus $I(t)$ recovers faster, preventing $I(t)$ from maintaining a small value for a long time and thus explaining the change in the PDFs. This difference between experiments and simulations might thus be due to more efficient recovery in experiment than in the model.



Fig. 4. $I(t)$ for small $k_{f}(\eta)$. (a, b) low $J: J=1.4 J_{t h} ;(\mathrm{c}, \mathrm{d})$ high $J$ : $J=2.4 J_{\text {th }}$. (a, c) simulation, $k_{f}=8 \mathrm{~ns}^{-1} ;(\mathrm{b}, \mathrm{d})$ experiment, $\eta=0.31$. The horizontal dashed line indicates $\langle I\rangle$.

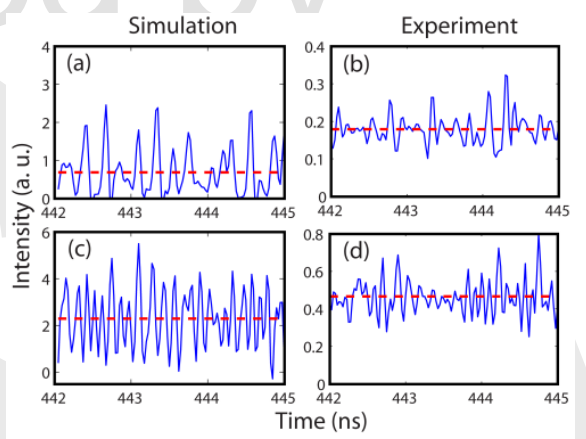

Fig. 5. $I(t)$ for large $k_{f}(\eta)$. (a, b) low $J: J=1.4 J_{t h} ;(\mathrm{c}, \mathrm{d})$ high $J$ : $J=2.4 J_{t h}$. (a, c) simulation, $k_{f}=25 \mathrm{~ns}^{-1} ;(\mathrm{b}, \mathrm{d})$ experiment, $\eta=1.0$.

Figures 5(c) and (d) also illustrate the strong difference in dispersion between theory and experiment; Fig. 5(c) shows much wider fluctuations around the mean than Fig. 5(d). Lower experimental dispersion of $I(t)$ is observed for all values of $J$ and $\eta\left(k_{f}\right)$, indicating stronger damping of oscillations between carrier and photon populations than accounted for by LK. We were not able to obtain a better fit between experimental and numerical PDFs by adjusting the LK parameters or by tuning the model itself, e.g., by introducing phenomenologically gain saturation with $I(t)[1,17,26]$ or by modeling the effect of noise with Langevin sources [1, 29] of typical intensities. Specifically, we find that even though the inclusion of gain saturation tends to decrease the residual pulsations present at low currents $\left(J \approx 1.4 J_{t h}\right.$ ), the shape of the PDFs does not become exponential at larger currents and the match between the theoretical and experimental ACFs worsens.

Another difference between theory and experiment is that the experimental PDFs are robust to changes in $J$ and $\eta$, while the LK-based PDFs vary with $J$, and to a smaller extent with $k_{f}$. To understand this, we compared the active EC modes (ECMs) predicted by the LK 
equations with the active modes from measured optical spectra. We see that, for moderate $k_{f}$, LK predicts that, as $J$ increases, the active modes undergo significant rightshift from a spectral range close to maximum-gain mode toward frequencies much closer to the minimumlinewidth mode (MLM) [1]. The difference in the dynamical properties of the selected ECMs (stability, basin) [11] may explain the variability of the PDFs. In contrast, experimental spectra show a relative invariance of the active modes, tending to be close to the MLM, for all $\eta$ and $J$ considered. This robust selection of active modes may contribute to the relative robustness of the experimental PDFs.

To conclude, we studied the statistics of $I(t)$ from an ECL in CC. Substantial agreement between theory and experiment was found for the autocovariance, both for $h$ and $\pi$ as functions of $\eta\left(k_{f}\right)$. This indicates good modeling by LK of linear correlations between successive dynamical states. Discrepancies are observed, however, in the PDFs. Theory is more skewed to low $I(t)$, but this diminishes for higher $J$, appearing increasingly Gaussian. While inclusion of noise or gain saturation slightly reduces the effect, it does not account for its size. We attribute this effect to residual intensity pulsations that are not present in experiments. Moreover, experimental PDFs for all parameters show robustly exponential-like shape, for all $\eta\left(k_{f}\right)$ and $J$, with smaller variance than simulations. Thus LK may overestimate, in the $\mathrm{CC}$ regime, the dispersion of the chaotic fluctuations with respect to $\langle I\rangle$ and the variability of the dynamics as a function of $\eta\left(k_{f}\right)$ and operating $J$.

The authors acknowledge the support of the Conseil Regional of Lorraine, GT-CNRS2958, and National Science Foundation grant ECCS-0925713. NL and WP also acknowledge the support of National Natural Science Foundation of China grant 61274042 and the funds for the Excellent Ph.D. Dissertation of Southwest Jiaotong University (2011).

\section{References}

1. M. C. Soriano, J. García-Ojalvo, C. R. Mirasso and I. Fischer, Rev. Mod. Phys. 85, 421 (2013).

2. A. Argyris, D. Syvrids, L. Larger, V. Annovazzi-Lodi, P. Colet, I. Fischer, J. Garcia-Ojalvo, C. R. Mirasso, L. Pesquera, and K. A. Shore, Nature 437, 343 (2005).

3. W. L. Zhang, W. Pan, B. Luo, X. H. Zou, M. Y. Wang, and Z. Zhou, Opt. Lett. 33, 237 (2008).

4. A. Uchida, K. Amano, M. Inoue, K. Hirano, S. Naito, H. Someya, I. Oowada, T. Kurashige, M. Shiki, S. Yoshimori, K. Yoshimura, and P. Davis, Nat. Photonics 2, 728 (2008).

5. I. Kanter, Y. Aviad, I. Reidler, E. Cohen, and M. Rosenbluh, Nat. Photonics 4, 58 (2010).

6. X. Z. Li and S. C. Chan, Opt. Lett. 37, 2163 (2012).

7. F. Y. Lin and J. M. Liu, IEEE J. Sel. Top. Quantum Electron. 10, 991 (2004).

8. L. Appeltant, M. C. Soriano, G. Van der Sande, J. Danckaert, S. Massar, J. Dambre, B. Schrauwen, C. R. Mirasso, and I. Fischer, Nat. Common. 2, 468 (2011).
9. D. Brunner, M. C. Soriano, C. R. Mirasso, and I. Fischer, Nat. Commun. 4, 1364 (2013).

10. J. Mørk, B. Tromborg, and J. Mark, IEEE J. Quantum Electron. 28, 93 (1992).

11. C. Masoller and N. B. Abraham, Phys. Rev. A 57, 1313 (1998).

12. B. Kim, N. Li, A. Locquet, and D. S. Citrin, Opt. Express 22, 2348 (2014)

13. R. Lang and K. Kobayashi, IEEE J. Quantum Electron. 16, 347 (1980).

14. A. Torcini, S. Barland, G. Giacomelli, and F. Marin, Phys. Rev. A 74, 063801 (2006).

15. T. S. Han, Information-Spectrum Methods in Information Theory (Springer-Verlag, 2002).

16. A. Locquet, "Chaos-Based Secure Optical Communications Using Semiconductor Lasers", in "Handbook of Information and Communication Security", edited by Mark Stamp and Peter Stavroulakis, Springer, pp. $451-478$ (2010).

17. D. W. Sukow, T. Heil, I. Fischer, A. Gavrielides, A. HohlAbiChedid, and W. Elsäßer, Phys. Rev. A 60, 667 (1999).

18. G. Huyet, J. K. White, A. J. Kent, S. P. Hegarty, J. V. Moloney, and J. G. McInerney, Phys. Rev. A 60, 1534 (1999).

19. G. Huyet S. Hegarty, M. Giudici, B. de Bruyn and J. G. McInerney, Europhys. Lett. 40, 619 (1997).

20. Y. Hong and K. A. Shore, Opt. Lett. 30, 3332 (2005).

21. J. Mørk, B. Tromborg, and P. L. Christiansen, IEEE J. Quantum Electron. 24, 123 (1988).

22. J. Sacher, W. Elsässer, and E. O. Göbel, Phys. Rev. Lett. 63, 2224 (1989).

23. T. Sano, Phys. Rev. A 50, 2719 (1994).

24. G. H. M. van Tartwijk, A. M. Levine, and D. Lenstra, IEEE J. Sel. Top. Quantum Electron. 1, 466 (1995).

25. I. Fischer, G. H. M. van Tartwijk, A. M. Levine, W. Elsässer, E. Göbel, and D. Lenstra, Phys. Rev. Lett. 76, 220 (1996).

26. M. Sciamanna, C. Masoller, F. Rogister, P. Mégret, N. B. Abraham, and M. Blondel, Phys. Rev. A 68, 015805 (2003).

27. A. Argyris, M. Boumpos, A. Bogris, and D. Syvridis, European Conference and Exhibition on Optical Communications (IET, 2013), pp. 1038-1040.

28. G. Aromataris and V. Annovazzi-Lodi, Photon. Technol. Lett. 24, 903 (2012).

29. D. Rontani, A. Locquet, M. Sciamanna, D. S. Citrin, and S. Ortin, IEEE J. Quantum Electron. 45, 879 (2009).

30. J. P. Toomey and D. M. Kane, Opt. Express 22, 1713 (2014).

31. S. Heiligenthal, T. Jüngling, O. D'Huys, D. A. ArroyoAlmanza, M. C. Soriano, I. Fischer, I. Kanter, and W. Kinzel, Phys. Rev. E 88, 012902 (2013).

32. X. Porte, M. C. Soriano, and I. Fischer, Phys. Rev. A 89, 023822 (2014).

33. N. Li, B. Kim, V. N. Chizhevsky, A. Locquet, M. Bloch, D. S. Citrin, and W. Pan, Opt. Express 22, 6634 (2014).

34. A. Wang, P. Li, J. Zhang, J. Zhang, L. Li and Y. Wang, Opt. Express 21, 20452 (2013).

35. N. Oliver, M. C. Soriano, D. W. Sukow, and I. Fischer, IEEE J. Quantum Electron. 49, 910 (2013).

36. J. Zhang, Y. Wang, M. Liu, L. Xue, P. Li, A. Wang, and M. Zhang, Opt. Express 20, 7496 (2012). 


\section{References}

1. M. C. Soriano, J. García-Ojalvo, C. R. Mirasso and I. Fischer, "Complex photonics: Dynamics and applications of delay-coupled semiconductor lasers," Rev. Mod. Phys. 85, 421 (2013).

2. A. Argyris, D. Syvrids, L. Larger, V. Annovazzi-Lodi, P. Colet, I. Fischer, J. Garcia-Ojalvo, C. R. Mirasso, L. Pesquera, and K. A. Shore, "Chaos-based communications at high bit rates using commercial fibre-optic links," Nature 438, 343 (2005).

3. W. L. Zhang, W. Pan, B. Luo, X. H. Zou, M. Y. Wang, and Z. Zhou, "Chaos synchronization communication using extremely unsymmetrical bidirectional injections," Opt. Lett. 33, 237 (2008).

4. A. Uchida, K. Amano, M. Inoue, K. Hirano, S. Naito, H. Someya, I. Oowada, T. Kurashige, M. Shiki, S. Yoshimori, K. Yoshimura, and P. Davis, "Fast physical random bit generation with chaotic semiconductor lasers," Nat. Photonics 2, 728 (2008).

5. I. Kanter, Y. Aviad, I. Reidler, E. Cohen, and M. Rosenbluh, "An optical ultrafast random number generator," Nat. Photonics 4, 58 (2010).

6. X. Z. Li and S. C. Chan, Opt. Lett. "Random bit generation using an optically injected semiconductor laser in chaos with oversampling," 37, 2163 (2012).

7. F. Y. Lin and J. M. Liu, "Chaotic lidar," IEEE J. Sel. Top. Quantum Electron. 10, 991 (2004).

8. L. Appeltant, M. C. Soriano, G. Van der Sande, J. Danckaert, S. Massar, J. Dambre, B. Schrauwen, C. R. Mirasso, and I. Fischer, "Information processing using a single dynamical node as complex system," Nat. Common. 2, 468 (2011)

9. D. Brunner, M. C. Soriano, C. R. Mirasso, and I. Fischer, "Parallel photonic information processing at gigabyte per second data rates using transient states," Nat. Commun. 4, 1364 (2013).

10. J. Mørk, B. Tromborg, and J. Mark, IEEE J. Quantum Electron. "Chaos in semiconductor lasers with optical feedback: theory and experiment," 28, 93 (1992).

11. C. Masoller and N. B. Abraham, "Stability and dynamical properties of the coexisting attractors of an external cavity semiconductor laser," Phys. Rev. A 57, 1313 (1998).

12. B. Kim, N. Li, A. Locquet, and D. S. Citrin, "Experimental bifurcation-cascade diagram of an external-cavity semiconductor laser," Opt. Express 22, 2348 (2014).

13. R. Lang and K. Kobayashi, "External optical feedback effects on semiconductor injection laser properties," IEEE J. Quantum Electron. 16, 347 (1980).

14. A. Torcini, S. Barland, G. Giacomelli, and F. Marin, "Low" frequency fluctuations in vertical cavity lasers: experiments versus Lang-Kobayashi dynamics," Phys. Rev. A 74, 063801 (2006).

15. T. S. Han, Information-Spectrum Methods in Information Theory (Springer-Verlag, 2002).

16. A. Locquet, "Chaos-Based Secure Optical Communications Using Semiconductor Lasers", in "Handbook of Information and Communication Security", edited by Mark Stamp and Peter Stavroulakis, Springer, pp. 451-478 (2010).

17. D. W. Sukow, T. Heil, I. Fischer, A. Gavrielides, A. HohlAbiChedid, and W. Elsäßer, "Picosecond intensity statistics of semiconductor lasers operating in the low-frequency fluctuation regime," Phys. Rev. A 60, 667 (1999).

18. G. Huyet, J. K. White, A. J. Kent, S. P. Hegarty, J. V. Moloney, and J. G. McInerney, "Dynamics of a semiconductor laser with optical feedback," Phys. Rev. A 60, 1534 (1999).
19. G. Huyet S. Hegarty, M. Giudici, B. de Bruyn and J. G. McInerney, "Statistical properties of the dynamics of semiconductor lasers with optical feedback," Europhys. Lett. 40, 619 (1997).

20. Y. Hong and K. A. Shore, "Statistical measures of the power dropout ratio in semiconductor lasers subject to optical feedback," Opt. Lett. 30, 3332 (2005).

21. J. Mørk, B. Tromborg, and P. L. Christiansen, "Bistability and low-frequency fluctuations in semiconductor lasers with optical feedback: a theoretical analysis," IEEE J. Quantum Electron. 24, 123 (1988).

22. J. Sacher, W. Elsässer, and E. O. Göbel, "Intermittency in the coherence collapse of a semiconductor laser with external feedback," Phys. Rev. Lett. 63, 2224 (1989).

23. T. Sano, "Antimode dynamics and chaotic itinerancy in the coherence collapse of semiconductor lasers with optical feedback," Phys. Rev. A 50, 2719 (1994).

24. G. H. M. van Tartwijk, A. M. Levine, and D. Lenstra, "Sisyphus effect in semiconductor lasers with optical feedback," IEEE J. Sel. Top. Quantum Electron. 1, 466 (1995).

25. I. Fischer, G. H. M. van Tartwijk, A. M. Levine, W. Elsässer, E. Göbel, and D. Lenstra, "Fast pulsing and chaotic itinerancy with a drift in the coherence collapse of semiconductor lasers," Phys. Rev. Lett. 76, 220 (1996).

26. M. Sciamanna, C. Masoller, F. Rogister, P. Mégret, N. B. Abraham, and M. Blondel, "Fast pulsing dynamics of a vertical-cavity surface-emitting laser operating in the low frequency fluctuation regime," Phys. Rev. A 68, 015805 (2003).

27. A. Argyris, M. Boumpos, A. Bogris, and D. Syvridis, "Statistical Properties of Broadband Chaotic Signals for Ultrafast True Random Bit Sequence Generation," European Conference and Exhibition on Optical Communications (IET, 2013), pp. 1038-1040.

28. G. Aromataris and V. Annovazzi-Lodi, "Error analysis of a digital message impaired by optical chaos," Photon. Technol. Lett. 24, 903 (2012).

29. D. Rontani, A. Locquet, M. Sciamanna, D. S. Citrin, and S. Ortin, "Time-delay identification in a chaotic semiconductor laser with optical feedback: a dynamical point of view," IEEE J. Quantum Electron. 45, 879 (2009).

30. J. P. Toomey and D. M. Kane, "Mapping the dynamic complexity of a semiconductor laser with optical feedback using permutation entropy," Opt. Express 22, 1713 (2014).

31. S. Heiligenthal, T. Jüngling, O. D’Huys, D. A. ArroyoAlmanza, M. C. Soriano, I. Fischer, I. Kanter, and W. Kinzel, "Strong and weak chaos in networks of semiconductor lasers with time-delayed couplings," Phys. Rev. E 88, 012902 (2013).

32. X. Porte, M. C. Soriano, and I. Fischer, "Similarity properties in the dynamics of delayed-feedback semiconductor lasers," Phys. Rev. A 89, 023822 (2014).

33. N. Li, B. Kim, V. N. Chizhevsky, A. Locquet, M. Bloch, D. S. Citrin, and W. Pan, "Two approaches for ultrafast random bit generation based on the chaotic dynamics of a semiconductor laser," Opt. Express 22, 6634 (2014).

34. A. Wang, P. Li, J. Zhang, J. Zhang, L. Li and Y. Wang, "4.5 Gbps high-speed real-time physical random bit generator," Opt. Express 21, 20452 (2013).

35. N. Oliver, M. C. Soriano, D. W. Sukow, and I. Fischer, "Fast random bit generation using a chaotic laser: approaching the information theoretic limit," IEEE J. Quantum Electron. 49, 910 (2013).

36. J. Zhang, Y. Wang, M. Liu, L. Xue, P. Li, A. Wang, and M. Zhang, "A robust random number generator based on differential comparison of chaotic laser signals," Opt. Express 20, 7496 (2012). 